\title{
A pathway to solving the Wi-Fi Tragedy of the Commons in apartment blocks
}

\author{
Frank den Hartog \\ DoVes Research \\ Canberra, Australia \\ frank.den.hartog@dovesresearch.net \\ Pia Kempker, Bert Boltjes \\ TNO \\ Den Haag, Netherlands \\ \{pia.kempker, bert.boltjes\}@tno.nl
}

\author{
Alessandro Raschella, Faycal Bouhafs \\ Department of Computer Science \\ Liverpool John Moores University \\ Liverpool, UK \\ \{A.Raschella, F.Bouhafs\} @ljmu.ac.uk
}

\author{
Mir Seyedebrahimi \\ Centre for Cloud Computing \\ Birmingham City University \\ Birmingham, UK \\ Mir.Seyedebrahimi@bcu.ac.uk
}

\begin{abstract}
Surprisingly little research has quantified the severity of Wi-Fi congestion in densely populated areas. We performed a high-fidelity 3D simulation of the performance of a realistic Wi-Fi deployment in a typical apartment block. Our results show that congestion leads to significant loss of performance, and that current channel selection procedures have only little effect. Also the strategy that is mostly applied today, i.e. to deploy additional repeaters and Access Points (APs), fails. As this is a typical example of the "Tragedy of the Commons", some form of collaboration between AP operators is needed to solve the problem. New channel selection algorithms that optimize Wi-Fi performance on a system level then become possible which, for instance, minimize the mutual interference impact on all APs involved. We validate that such an algorithm indeed leads to an optimized as well as fair assignment, which is a necessary first step towards solving the Tragedy.
\end{abstract}

Keywords- License-free spectrum; spectrum management; spectrum efficiency.

\section{INTRODUCTION}

Different studies, e.g. [1], show that the scarcity of radio frequency spectrum is a limiting factor for the development of wireless broadband networks. Wi-Fi forms a special case as it uses unlicensed spectrum. The overwhelming success of tablets and smart phones is a key factor amongst others driving the dense deployment of Wi-Fi Access Points (APs) in practically all homes with an Internet connection. The drawback of this dense deployment is the potential for co-channel and adjacent channel interference with nearby devices.

Although there is plenty of anecdotal evidence that many users are experiencing performance issues in such cases [2], surprisingly little research has been done so far to quantify the pervasiveness and severity of the problem [3]. It is therefore too early for blanket solutions that require large investments and changes in regulation and the operators' networks [3,4]. Besides, given the relatively short range of most techniques working in the unlicensed bands, the problem is usually confined to specific locations. Solutions should therefore be cost-effective and locally implementable.

The design of such solutions is not trivial. To understand the issue, we need to realize that unlicensed spectrum is a "commons" in economic terms, and performance loss can be seen as a typical example of the "Tragedy of the Commons" $[4,5]$. This is particularly the case in apartment blocks, where Wi-Fi networks are managed by more than one operator, the operators often being the end users themselves. Therefore, as others concluded before [3], the issue must be treated as a joint engineering, regulatory, and economic problem.

In previous work we have investigated the economic and regulatory aspects of $\mathrm{Wi}-\mathrm{Fi}$ over-congestion in apartment blocks. By applying game theory [6], we showed that solving the Tragedy is only possible by unselfish collaboration between the players, which in this case means, some form of collaboration between the APs (and their operators) on a system level. However, the algorithms and platforms for negotiation and execution of inter-AP coordination in a multiple-operator environment do not yet exist.

In this article we first evidence the issue in a typical apartment block by means of OPNET simulations of Wi-Fi in the $2.4 \mathrm{GHz}$ band. We found that the only way to obtain realistic results is by modeling the use case in $3 \mathrm{D}$, which has not been done before. We then show that a simple intuitive agreement among the AP operators regarding who is using which channel already leads to a significant improvement of the overall performance. However, as such agreement does not guarantee a fair distribution of resources among the players, it has a high chance of some players defecting the collaboration. We, therefore, applied and validated a novel algorithm which assigns the channels such that the performance in every apartment is optimized as well as equal (where we for now assume that "equal = fair") [7]. We conclude with a discussion on the implications of our results for the Wi-Fi industry. 


\section{STATE OF THE ART}

\section{A. Congestion in Unlicensed Spectrum}

In 2013, Ozyagci et al [2] provided an overview of the anecdotal evidence that exists for Wi-Fi congestion in densely populated places. By simulation in OPNET, the authors then investigated the effect of adding APs to a confined 2D indoor space on the total capacity of the system. The attenuation of the Wi-Fi signal by internal walls is emulated by (unrealistically) adding randomly positioned walls with varying densities. Ozyagci's work emphasizes the need to distinguish three different causes of performance loss:

- Interference means that $\mathrm{Wi}-\mathrm{Fi}$ network nodes receive signals they cannot recognize as Wi-Fi traffic. It is typically caused by Industrial, Scientific, and Medical (ISM) devices in the neighborhood, or devices using a different communication protocol such as Bluetooth or Zigbee, or Wi-Fi devices emitting on different but overlapping channels (adjacent channel interference).

- Congestion means that, given the interference and noise levels the network nodes are experiencing on the physical layer, the network is carrying the maximum amount of data it can handle. Wi-Fi's Medium Access Control (MAC) protocol still allows additional APs to transmit, due to its random-back-off mechanism, but ultimately the total capacity will be shared with the other APs, and the achievable throughput of every individual AP will go down, even though the total capacity may have increased.

- Over-congestion is observed when adding APs to an already congested system leads to a decrease of the total system capacity: the number of packet collisions is so large that the system's capacity is largely consumed by control traffic.

As the Tragedy of the Commons is defined by a situation where individual users acting independently according to their own self-interest behave contrary to the common good of all users, by depleting or spoiling that resource through their collective action, this only applies to over-congested systems.

In 2014, Saber Kamooshi [8] modelled the impact of a rather unrealistic 3D indoor environment on the performance of ultradense wireless networks. The model is validated with Monte Carlo simulations in MatLab. Interestingly, Kamooshi found that floor losses have a significant impact on the Wi-Fi performance. Like Ozyagci, he also found that increasing the number of APs may lead to a decrease in $\mathrm{Wi}-\mathrm{Fi}$ performance.

\section{B. Channel assignment algorithms}

In addressing the interference problem, most Wi-Fi APs currently in the market adopt a per-cell dynamic channel assignment approach such as Least Congested Channel (LCC) [9]. In LCC, each AP scans the radio spectrum at the physical layer, and then selects the least congested channel, either being the channel with the lowest number of connected APs, or the one with the lowest accumulated value of sensed powers [10]. In practice, apparently, the result is often unsatisfactory. Besides, this approach does not temper or punish the greedy behavior of players, and will therefore not solve the Tragedy.

As said before, the Tragedy can only be solved by having the players, i.e. the AP operators of APs that are within each other's range, accept that they need to collaborate in an unselfish way. To achieve this, we propose that AP operators delegate the configuration of their APs to a central controller that executes a commonly agreed-upon resource sharing policy [7]. Such a system enables the execution of channel assignment algorithms that find their basis in the vast literature on cellular [11] and cognitive [12] networks.

In [7], we designed such an algorithm, where we introduced the concept of interference impact. Interference impact is the contribution that each individual AP has in generating interference at various locations throughout the network. These locations are chosen to be the other APs' locations as the central controller has easy access to the SINR values observed by these APs, and also has full control over the APs' settings. The algorithm consequently tries to minimize the accumulated interference impact over the available channels, given the network topology. In contrast to current enterprise Wi-Fi networks, we here make full use of our knowledge of the APs' locations to find a global performance optimum, whereas APs in enterprise networks merely try to choose a channel based on local measurements of the accumulative interference signal from different sources.

\section{THE APARTMENT BLOCK USE CASE AND MODEL}

\section{A. Use case}

To model a typical apartments block use case we took the apartment building De Baron in Zoetermeer (Netherlands) as an example. The building has four floors, four wings and a total of 75 apartments. For the scope of the simulation, only one $25 \times 13$ $\mathrm{m}$ sized wing is considered. The floor plan (see Fig. 1) is the same for all floors. Each floor is composed of four large singlebedroom apartments and one smaller studio. We used the following attenuation values for perpendicular penetration $[13,14]$ :

- Thick walls - high density, of $22.5 \mathrm{~cm}$ thickness, surrounding each apartment: $18 \mathrm{~dB}$;

- Thin walls - low density, of $11 \mathrm{~cm}$ thickness, all apartment internal walls: $5.5 \mathrm{~dB}$;

- Floors - high density, between the different levels of the wing: $25 \mathrm{~dB}$;

- Air-between the devices with no obstacles: $1 \mathrm{~dB} / \mathrm{m}$.

We assume that the APs are situated within the utility closets in each of the apartments, that there are a maximum of three devices actively using $\mathrm{Wi}-\mathrm{Fi}$ at any given time in each of the large apartments (e.g. a laptop, a tablet and a phone), and two devices in the small studios. The utility closets are next to the front doors that open to the corridor. In total, the model thus consists of 76 (19 per floor, 4 identical floors) Wi-Fi devices being active at the simulated moment. This could be, for instance, peak time during an evening. 


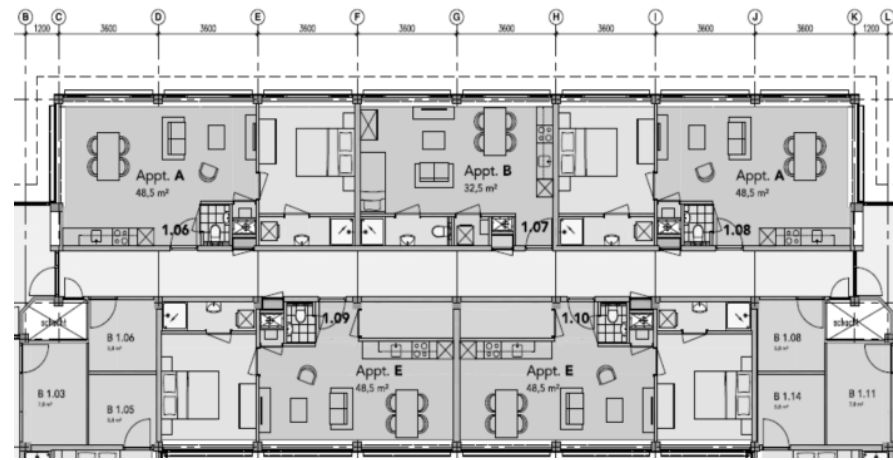

Fig. 1. Floor plan of one of the floors in one of the wings of the apartment block as used in our simulations.

\section{B. The OPNET Model}

The OPNET Modeler (version 17.5) and the OPNET Wireless Suite were used as a basis for the simulations. To our knowledge, this is the first time that $3 \mathrm{D}$ indoor $\mathrm{Wi}-\mathrm{Fi}$ performance is modelled in OPNET. For this, we modified the OPNET WLAN model to import and use matrices for the attenuation of radio signals between pairs of Wi-Fi nodes, and to automate the configuration of the nodes for sets of simulation runs. Attenuation matrices contain the signal attenuation between each node pair, and were pre-computed and fixed during the simulations. Non-perpendicular penetration is taken into account by measuring the thickness of the air, walls, and floors along the straight line between transmitter and receiver. The attenuation matrix file is read once by the NodeConfig module, which is added to the standard WLAN node model.

The external parameters, like the matrix, are declared in an added header file so they can be accessed once and then read throughout the code of the model. The matrix is parsed to a symmetric two-dimensional matrix in which each element contains the attenuation between the nodes in each node pair. This matrix is used by the closure transmission radio pipeline stage that determines which receiver receives the radio packet that has to be transmitted, and its signal strength. A special kernel was added to this pipeline stage that determines the IDs of the transmitter and the receiver. It uses these IDs to determine the cell in the matrix to retrieve the attenuation and compute the signal strength at the receiver.

Various parameters are defined through attributes added to the node model. These attributes, shown in Table 1, are read by the NodeConfig module. Note that isotropic antennas with a gain of $0 \mathrm{dBi}$ are used: the attribute at the top of the list in Table 1 is set to zero for all nodes when the simulation starts. The process in this module has been given highest priority. Therefore it will be the first process to run when the simulation starts. Promoting key attributes such as the transmit power and the file name with the attenuation matrix, enables one to define sets of simulation runs in which a different attribute value is used for each run. This also speeds up the analysis and comparison of statistics collected during the runs.

Here we only present simulation results for the $2.4 \mathrm{GHz}$ band, assuming that everybody uses IEEE $802.11 \mathrm{~g}$ or IEEE 802.11 n, operating without MIMO, with $20 \mathrm{MHz}$ bands, and 54 $\mathrm{Mb} / \mathrm{s}$ transmission rate without automatic rate adaptation, and sends the maximum amount of traffic from the AP to the devices. The only variable parameter in the simulation is the selected channel. The transmission power is fixed at $100 \mathrm{~mW}$, and the packet reception-power threshold, i.e. the receiver sensitivity, is set at $-95 \mathrm{dBm}[15]$.

TABLE I. ATTRIBUTES ADDED TO THE WLAN NODE MODEL.

\begin{tabular}{|c|c|}
\hline NodeConfig Attributes & State \\
\hline Antenna Gain Relative to $60 \mathrm{MHz}$ value (in $\mathrm{dB}$ ) & Promoted \\
\hline File_Name_of_the_Radio_Attenuation_Matrix & Promoted \\
\hline Node ID & Promoted \\
\hline Transmit Power (Watt) & Promoted \\
\hline NodeConFig.Overall Additional Pathloss & Promoted \\
\hline NodeConFig.Pathloss Closure Threshold (dB) & Promoted \\
\hline NodeConFig.Power Aware Closure & Promoted \\
\hline NodeConFig.Use_number_at_the_end_of_the_node_name & Promoted \\
\hline Destination Address & Broadcast \\
\hline Traffic Type of Service & Best Effort \\
\hline
\end{tabular}

We generate traffic flows of varying lengths, exponentially distributed with an average flow length of $3 \mathrm{~s}$. The downtime between traffic flows is also exponentially distributed, with an average of $0.01 \mathrm{~s}$. Each traffic flow consists of packets that are generated according to a Poisson process with an average interarrival time of $0.00039 \mathrm{~s}$. The packet size is 1024 bytes per packet. This results in a traffic flow averaging $21 \mathrm{Mb} / \mathrm{s}$, which is close to the maximum throughput achievable in IEEE $802.11 \mathrm{~g}$ [16], and we indeed observe packet loss when we send traffic at a higher rate. Using maximum traffic assumes a worst case scenario, but it makes the results easier to interpret. Traffic is broadcasted from the AP to the different devices.

Every simulation is run 3 times, for seeds 11, 22, 33, and the results (which were always within $0.1 \%$ of each other) were averaged. The simulation time is 1 minute, although we found that the results always converge after a few seconds. On our hardware, a single simulation typically takes 15 minutes, which scales quadratically with the number of simulated devices.

\section{RESULTS}

\section{A. All APs on channel 1}

In our first experiment, we assign all channels to " 1 ". This creates a baseline for our results with distributed channel assignments, and aids with validating our set up. Having all APs operating on channel 1 is not a typical use case, but neither is it unrealistic: many of the older APs have channel 1 set by default and have a very basic channel selection algorithm (if at all), often converging to channel 1 [4]. Most people do not change the default settings of their AP.

We also vary the isolation between the apartments by adding an additional box (wall, floor and ceiling) around each apartment. The attenuation losses caused by these additional walls are varied between $0 \mathrm{~dB}$ (i.e. no additional attenuation) to $100 \mathrm{~dB}$. Fig. $2 \mathrm{a}$ and $2 \mathrm{~b}$ show the average throughput per device per apartment in $\mathrm{Mb} / \mathrm{s}$ for $0 \mathrm{~dB}$ and $100 \mathrm{~dB}$ attenuation respectively, for every set of 5 apartments on the four different floors. The grey shading is a subjective index representing "good" (white), "fair" (grey), or "bad" (black). The white dumbbell shapes represent the corridors. 


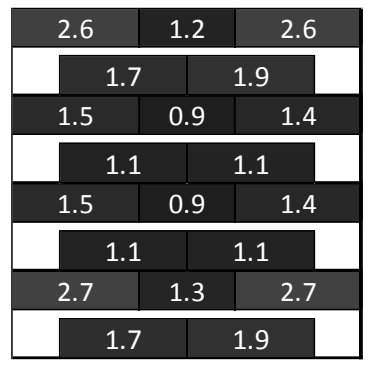

a)

\begin{tabular}{|c|c|c|}
\hline 20.6 & 20.6 & 20.6 \\
\hline \hline 20.6 & \multicolumn{2}{|c|}{20.6} \\
\hline 20.6 & 20.6 & 20.6 \\
\hline \multicolumn{2}{|c|}{20.6} & \multicolumn{2}{|c|}{20.6} \\
\hline 20.6 & 20.6 & 20.6 \\
\hline 20.6 & \multicolumn{2}{|c|}{20.6} \\
\hline 20.6 & 20.6 & 20.6 \\
\hline \multicolumn{2}{|c|}{20.6} & 20.6 \\
\hline
\end{tabular}

b)
Fig. 2. Average throughput per device per apartment in $\mathrm{Mb} / \mathrm{s}$ with all APs operating on channel 1 , with a) $0 \mathrm{~dB}$, and b) $100 \mathrm{~dB}$ additional isolation between the apartments.

We conclude that in the normal case (with $0 \mathrm{~dB}$ attenuation between the apartments), all apartments have their Wi-Fi performing on only $\sim 5-10 \%$ of their maximum capacity $(21 \mathrm{Mb} / \mathrm{s})$ if all APs operate on the same channel. This proves that congestion can indeed be a problem in current deployments. With an attenuation loss of $100 \mathrm{~dB}$ between neighboring apartments we achieve the throughputs we expect without interference, which validates our model.

Fig. 3 shows the results for $30 \mathrm{~dB}$ (a) and $60 \mathrm{~dB}$ (b) attenuation. Like in most other cases, we find that the apartments in the middle of the block experience the worst performance. This confirms the need of performing 3D modeling instead of $2 \mathrm{D}$ to obtain realistic results [8].

\begin{tabular}{|l|l|l|}
\hline 6.6 & 3.1 & 7.7 \\
\hline \multicolumn{2}{|c|}{7.7} & \multicolumn{2}{|c|}{5.2} \\
\hline 3.3 & 1.5 & 4.1 \\
\hline \multicolumn{2}{|c|}{3.6} & \multicolumn{2}{|c|}{2.3} \\
\hline 3.2 & 1.4 & 3.9 \\
\hline \multicolumn{2}{|c|}{3.7} & 2.3 \\
\hline 6.7 & 3.2 & 7.9 \\
\hline \multicolumn{2}{|c|}{7.7} & \multicolumn{2}{|c|}{5.2} \\
\hline
\end{tabular}

a)

\begin{tabular}{|c|c|c|}
\hline 11.2 & 9.4 & 11.2 \\
\hline \multicolumn{2}{|c|}{14.0} & 14.0 \\
\hline 9.9 & 10.3 & 9.9 \\
\hline \multicolumn{2}{|c|}{6.9} & 6.9 \\
\hline 9.7 & 10.4 & 9.7 \\
\hline \multicolumn{2}{|c|}{6.9} & 6.8 \\
\hline 11.4 & 9.3 & 11.4 \\
\hline \multicolumn{2}{|c|}{14.0} & 14.0 \\
\hline
\end{tabular}

b)
Fig. 3. Average throughput per device per apartment in $\mathrm{Mb} / \mathrm{s}$ with all APs operating on channel 1 , with a) $30 \mathrm{~dB}$, and b) $60 \mathrm{~dB}$ additional isolation between the apartments.

This is further illustrated by Fig. 4. Here we assumed impenetrable floors (100 $\mathrm{dB}$ attenuation) and varied the attenuation between the apartments. Now, every floor performs the same. Furthermore, the results are comparable to what has been found in [2] $\left(0,015 \mathrm{AP} / \mathrm{m}^{2}\right.$, strong attenuation, 5.3-8.0 Mb/s/AP), again validating our model.

\section{B. A typical distributed channel assignment}

Fig. 5 shows the results for a channel assignment typical for most apartment blocks today [4]: a number of people manually configure their APs such that they operate on a "free channel". Now we find that per receiving device, the average throughput over all apartments is $3.3 \mathrm{Mb} / \mathrm{s}$. This is only marginally better than the $1.6 \mathrm{Mb} / \mathrm{s}$ we found if all channels are assigned to 1 , given the $21 \mathrm{Mb} / \mathrm{s}$ that should be ideally achievable. From this we conclude that trying to "find a free channel" does not solve much, because adjacent channel interference has a devastating effect on the overall performance.

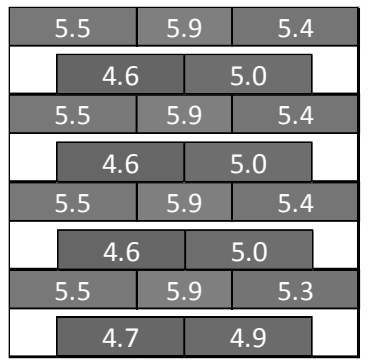

a)

\begin{tabular}{|c|c|c|}
\hline 8.3 & 4.3 & 10.0 \\
\hline \multicolumn{2}{|c|}{10.4} & 7.1 \\
\hline 8.3 & 4.3 & 10.1 \\
\hline \multicolumn{2}{|c|}{10.5} & 7.1 \\
\hline 8.3 & 4.3 & 10.0 \\
\hline \multicolumn{2}{|c|}{10.4} & 7.1 \\
\hline 8.3 & 4.3 & 10.0 \\
\hline & & 7.1 \\
\hline
\end{tabular}

b)
Fig. 4. Average throughput per device per apartment in Mb/s with all APs operating on channel 1 , with $100 \mathrm{~dB}$ additional isolation between floors, and a) $0 \mathrm{~dB}$, and b) $30 \mathrm{~dB}$ additional isolation between the apartments.

\begin{tabular}{|l|l|l|l|}
\hline 1 & 6 & 3 \\
\hline & \multicolumn{2}{|c|}{11} & \multicolumn{2}{c|}{4} \\
\hline 6 & \multicolumn{2}{|c|}{11} & 1 \\
\hline \multicolumn{2}{|c|}{4} & \multicolumn{2}{|c|}{1} \\
\hline 1 & 9 & 11 \\
\hline \multicolumn{2}{|c|}{11} & \multicolumn{2}{c|}{1} \\
\hline \multicolumn{2}{|c|}{11} & 11 \\
\hline 6 & \multicolumn{2}{|c|}{8} \\
\hline \multicolumn{2}{|c|}{6} & \multicolumn{2}{|c|}{8} \\
\hline
\end{tabular}

a)

\begin{tabular}{|c|c|c|}
\hline 3.5 & 3.7 & 7.6 \\
\hline \multicolumn{2}{|c|}{2.4} & 0.1 \\
\hline 4.4 & 3.7 & 4.7 \\
\hline \multicolumn{2}{|c|}{0.1} & 2.7 \\
\hline 3.6 & 3.8 & 2.6 \\
\hline \multicolumn{2}{|c|}{2.7} & 2.9 \\
\hline 4.4 & 3.5 & 2.8 \\
\hline 3. & & 4.4 \\
\hline
\end{tabular}

b)
Fig. 5. a) Typical channel assignment one finds in most apartment blocks. b) Average throughput per device per apartment in $\mathrm{Mb} / \mathrm{s}$ with the channel assignment of a).

We repeated the simulations for an increasing number of APs being switched off, in random order. The dashed curve in Fig. 6 shows the average throughput per apartment vs the number of APs being switched off. The results differ for the different orders in which the APs are switched off (we tried 10 different orders), and the variation in results is represented by the error bars. From these results we conclude that the maximum capacity of the system is already reached with only 10 of the 20 APs still switched on. The other 10 APs are basically a waste of money. Said otherwise, the strategy that is mostly propagated today by operators in case of performance issues, i.e. to deploy additional repeaters and access points (APs), will fail in densely built environments.

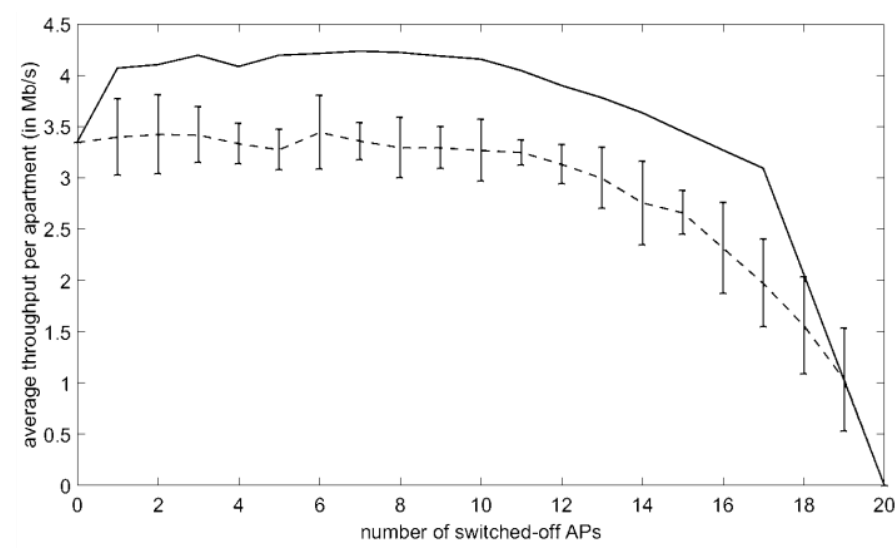

Fig. 6. Average throughput over all apartments in $\mathrm{Mb} / \mathrm{s}$ as a function of the number of APs being switched off, for the channel assignment of Fig. 5a).

The dashed curve also seems to indicate a slight increase in capacity when switching off the first three APs, suggesting that 
the system is in a state of over-congestion, and thus evidencing the Tragedy. This effect is emphasized by the solid curve, which is obtained by switching APs on one by one (so building op the curve from the right), where every AP to be switched on is chosen such that the total capacity is maximized. Congestionfree performance is observed with the first three APs switched on (average congestion-free throughput = \#APs x $21 \mathrm{Mb} / \mathrm{s} / 20$ apartments). The maximum system capacity is reached after 13 APs have been switched on, after which the capacity rapidly decreases. It needs to be remarked that, here, the decrease in performance is due to a mix of over-congestion and adjacent channel interference. But as the adjacent channel interference is caused by users willingly selecting another channel in order to maximize their performance, this behavior also contributes to the Tragedy.

We repeated the simulations assuming that all APs choose their own channel using the LCC mechanism [9], selecting the channel with the lowest value of sensed power [10]. This, however, leads to a very similar channel assignment as shown in Fig. 5 a), and to similar performance results (not shown).

\section{A Typical Mutually Agreed Channel Assignment}

We now imagine a case where the residents are well aware of the problem and try to collaborate and "manually" agree on a channel assignment: they eliminate adjacent channel interference by only using channels 1,6 , and 11 , and try to minimize co-channel interference. The resulting channel assignment could very well look like the one shown in Fig. 7 a): no closest neighbors (i.e. the apartments directly above and below, the apartments to the left and right, and the apartments directly opposite) share a channel. Fig. 7 b) shows the resulting Wi-Fi performance. The average throughput over all apartments is now $5.42 \mathrm{Mb} / \mathrm{s}$, which is significantly more than what we found for the uncoordinated cases in the previous section, but still only $25 \%$ of the maximum achievable throughput. Besides, switching off the APs in the middle two small apartments (row 3 , column 2, and row 5, column 2) leads to an overall average throughput of $5.47 \mathrm{Mb} / \mathrm{s}$, indicating that the system is still in an over-congested state.

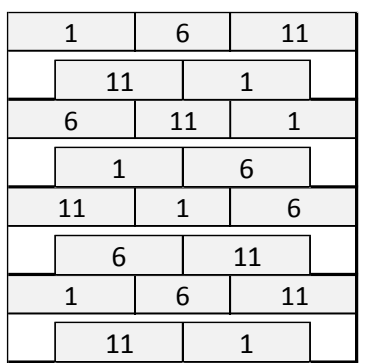

a)

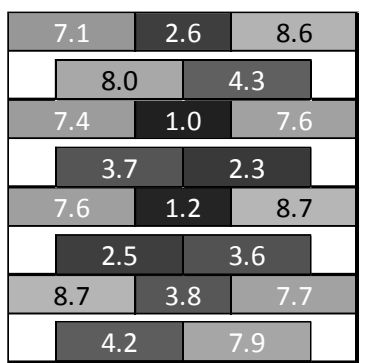

b)
Fig. 7. a) Typical mutually agreed channel assignment one may find in an apartment block. b) Average throughput per device per apartment in Mb/s with the channel assignment of a).

Also, for some apartments the results are worse compared to the situation shown in Fig. 5b. These residents may therefore choose to defect the collaboration, and then decide to select a "free channel" (2-5 or 7-10) anyway. What happens next is difficult to predict. The defectors may decide to rejoin the collaboration ("wasn't so bad after all"), or other tenants may decide to leave the collaboration also. This is for further study.

We here propose to avoid this scenario from happening by applying the interference mitigating algorithm as proposed in [7] which assigns the channels such that the Wi-Fi performance in every apartment is optimized as well as equal. Assuming that this will be considered as "fair", it will limit the chance of players defecting, even if their performance is less than in the uncoordinated scenario. By the way, the algorithm also allows for optimization over resource distributions other than "equal".

\section{Minimizing the Interference Impact}

Applying [7] to our use case yields the channel assignment and performance results as shown in Fig. 8. Again, for some apartments the results are worse compared to the situation shown in Fig. 5 b). These residents may therefore choose not to collaborate. However, as the new results now seem "fair", and the performance is still quite reasonable, it will be more likely that everybody will continue the collaboration anyway. The average throughput over all apartments is now $4.60 \mathrm{Mb} / \mathrm{s}$, which is only marginally less than obtained in Fig. 7.

\begin{tabular}{|c|c|c|c|}
\hline 1 & & 6 & 1 \\
\hline & 11 & & 1 \\
\hline 6 & & 11 & 1 \\
\hline & 6 & & 6 \\
\hline 1 & & 11 & 11 \\
\hline & 11 & & 1 \\
\hline 6 & & 11 & 11 \\
\hline & 6 & & 1 \\
\hline
\end{tabular}

a)

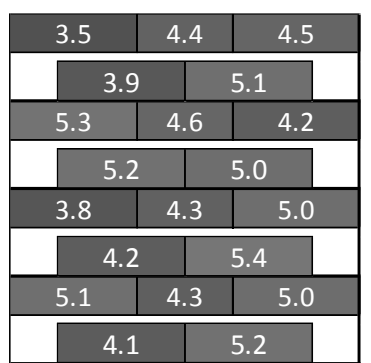

b)
Fig. 8. a) Channel assignment resulting from our fair interference impact distribution algorithm b) Average throughput per device per apartment in $\mathrm{Mb} / \mathrm{s}$ with the channel assignment of a).

Again we switched off one AP after the other, in random order, and repeated the simulation. Fig. 9 shows the average throughput over all apartments versus the number of APs being switched off. The average throughput goes down continuously, proving that the system is not over-congested anymore. Furthermore, the error bars are much smaller than in Fig. 6, showing that all APs are indeed impacting each other equally.

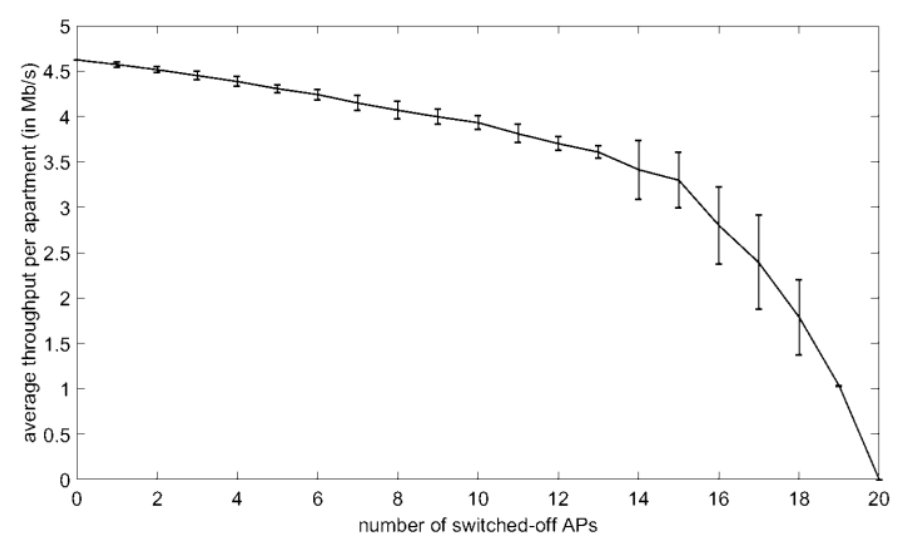

Fig. 9. Average throughput over all apartments in $\mathrm{Mb} / \mathrm{s}$ as a function of the number of APs being switched off, for the channel assignment of Fig. 8 a). 


\section{DISCUSSION}

The reasons why we model with $\mathrm{Wi}-\mathrm{Fi} \mathrm{g}$ are the following:

- Wi-Fi g is still prevalent in current homes, and is expected to stay for a long time to come (as even $\mathrm{Wi}-\mathrm{Fi}$ $\mathrm{b}$ is still around today).

- Wi-Fi n and -ac scale down to -g at low SINRs, which is the area of interest of this study; novel techniques to enhance capacity such as Multiple Input Multiple Output (MIMO) are not effective at low SINR.

- Beamforming is an effective way of boosting signal strength at the targeted receiver, and reducing interference to other devices, but in a densely deployed network the APs have to apply it in many directions at the same time, basically reducing its effectivity.

- Channel bonding is only aggravating the issue, as it just serves the greediness of individual players.

We also realize that the solution space we are describing is rather contrary to where the Wi-Fi industry is going today, and will therefore not be enthusiastically embraced any time soon. However, in this and other papers we promoted the notion of the Wi-Fi Tragedy of the Commons to a scientific fact, and it is a fact as well that it can only be solved by collaboration. Any other approach is only aggravating the issue, or at best postponing it for a few years. The only alternative is making new spectrum available faster than the desire for resources is growing. Reference [4] shows that this is not what is happening today: the first signs of congestion in the $5 \mathrm{GHz}$ band are already being observed.

\section{CONCLUSIONS}

We performed the first 3D high-fidelity simulations of the performance of $\mathrm{Wi}-\mathrm{Fi}$ in a dense apartment block deployment use case. We proved that inter-floor interference can have a significant effect on the Wi-Fi performance, which invalidates any previous high-fidelity 2D modeling in the literature.

The simulations clearly indicate that over-congestion is already a problem in today's dense apartment blocks. As a consequence, operators and apartment dwellers should immediately stop trying to solve their performance issues by just adding APs and repeaters. Current adaptive but noncollaborative channel selection procedures have only little effect. AP operators first need to agree that collaborative channel assignment is a necessity, in a way that avoids a grossly unfair distribution of resources over the individual apartments. This issue can be solved by applying a new channel selection algorithm which minimizes the mutual interference impact on all access points involved.

Future work includes:

- Further simulation for other Wi-Fi versions and other technologies using unlicensed bands, such as Zigbee, Bluetooth, Long Term Evolution (LTE) Unlicensed, and LoRa, including mixed-technology use cases,
- Investigating collaboration schemes other than joint channel selection, including transmit power control, horizontal handover, and vertical handover,

- Performing real-life measurements in dense apartment blocks like the one simulated here.

\section{ACKNOWLEDGEMENT}

This work has been carried out in the framework of the Wi-5 project, which is partly funded by the European Union's Horizon 2020 Research and Innovation Program under Grant Agreement no. 644262 .

\section{REFERENCES}

[1] I. Al-Alawi, "WiFi Technology: Future Market Challenges and Opportunities," Journal of Computer Science, vol. 2, no. 1, pp. 13-18, Jan. 2006.

[2] A. Ozyagci, K. W. Sung, and J. Zander, "Effect of propagation environment on area throughput of dense WLAN deployments," 2013 IEEE Globecom Workshops (GC Wkshps), 2013.

[3] J.P. De Vries, L. Simic, A. Achtzehn, M. Petrova, and P Mähönen, "The emperor has no problem: Is wi-fi spectrum really congested?," 41st Research Conference on Communication, Information and Internet Policy (TPRC 41). Sep. 2013.

[4] F. den Hartog and J. de Nijs, "The role of regulation in preventing Wi-Fi over-congestion in densely populated areas," Australian Journal of Telecommunications and the Digital Economy, vol. 5, no. 2, pp. 1-16, Jun. 2017.

[5] P.J. Weiser and D.N. Hatfield, "Policing the spectrum commons," Fordham L. Rev., Vol. 74, p. 663, 2005.

[6] M.P.P. van Heesch, "Combined Cooperation and Non-Cooperation for Channel Allocation and Transmission Power Control," Master's Thesis, Tilburg University, 2016.

[7] M. Seyedebrahimi, F. Bouhafs, A. Raschella, M. Mackay, and Q. Shi, "Fine-Grained Radio Resource Management to Control Interference in Dense Wi-Fi Networks," 2017 IEEE Wireless Communications and Networking Conference (WCNC), 2017.

[8] S. Kamooshi, "Impact of Three-Dimensional Indoor Environment on the Performance of Ultra-Dense Wireless Networks," Master's Thesis, KTH Royal Institute of Technology, 2014.

[9] M. Achanta, "Method and Apparatus for Least Congested Channel Scan for Wireless Access Points," US Patent No. 20060072602, Apr. 2006.

[10] S. Chieochan, E. Hossain, and J. Diamond, "Channel assignment schemes for infrastructure-based 802.11 WLANs: A survey," IEEE Communications Surveys \& Tutorials, vol. 12, no. 1, pp. 124-136, 2010.

[11] H. W. Cho, S. J. Bae, H. Choo, and M. Y. Chung, "Implementation of WLAN Connection Management Schemes in Heterogeneous Network Environments with WLAN and Cellular Networks," Computational Science and Its Applications - ICCSA 2011 Lecture Notes in Computer Science, pp. 32-43, 2011.

[12] E. Ahmed, A. Gani, S. Abolfazli, L. J. Yao, and S. U. Khan, "Channel Assignment Algorithms in Cognitive Radio Networks: Taxonomy, Open Issues, and Challenges," IEEE Communications Surveys \& Tutorials, vol. 18, no. 1, pp. 795-823, 2016.

[13] R. Rudd, K. Craig, M. Ganley, and R. Hartless, "Building Materials and Propagation," Final Report, Ofcom, 2604/BMEM/R/3/2.0, 2014.

[14] 3Com Wireless Antennas Guide. lojati.com.br/Customer/Datasheets/ 3CWE598.pdf, 2015.

[15] B. Kellogg, V. Talla, J. R. Smith, and S. Gollakot, "Passive Wi-Fi," GetMobile: Mobile Computing and Communications, vol. 20, no. 3, pp. 38-41, May 2017.

[16] D. Vassis, G. Kormentzas, A. Rouskas, and I. Maglogiannis, "The IEEE 802.11g standard for high data rate WLANs," IEEE Network, vol. 19, no. 3 , pp. 21-26, 2005. 